\title{
A novel approach to 2D drawings-based reconstruction of 3D building digital models
}

\author{
L. Gimenez ${ }^{1}$, S. Robert ${ }^{1}$, F. Suard ${ }^{1} \&$ K. Zreik ${ }^{2}$ \\ ${ }^{1}$ CEA, LIST, F-91191 Gif-sur-Yvette, France \\ ${ }^{2}$ University Paris 8, UFR6, Hypermedia Department, France
}

\begin{abstract}
There is an opportunity to significantly impact building energy efficiency through BIM-enabled, simulation-intensive, cost-effective renovation actions. One major hurdle however is the lack of 3D digital models for the majority of existing buildings. Cost-effective and widely applicable methods and tools are required for the reconstruction of 3D digital models from available information. In this scope, this paper presents ongoing research about the development of tools for semi-automated 3D building model generation from 2D scanned plans. More specifically, the paper focuses on the description of an innovative reconstruction process based on a combination of automated processing and punctual, softwareassisted, user intervention. The paper also gives an account of the experiments lead with a research prototype, tested on 90 real architectural floor plans. The results are encouraging and suggest that the mix of software-assistance and focused human intervention may be the best trade-off to upgrade the quality of the generated models and to achieve cost-effectiveness.

Keywords: Building Information Modeling (BIM), image processing, 3D building models, $3 D$ reconstruction, model checking.
\end{abstract}

\section{Introduction}

It is widely acknowledged that one of the most important challenges in the construction sector is to improve building energy efficiency. This particularly applies to Europe where more than $40 \%$ of the residential housing stock was built before 1960 and requires heavy renovation. At the same time, AEC (Architecture, Engineering and Construction) practices and tools have evolved towards more effective, ICT-intensive, BIM (Building Information Model)-based, digital design. 
Therefore, this seems to be the right time to significantly enhance building energy efficiency, through simulation-intensive, cost-effective renovation actions [1].

A large set of techniques already exist to create 3D models of existing buildings [2], e.g. photogrammetry and laser scanning. As shown by a recent review [3], the selection of the most adequate method will highly depend on the specifics of the project targeted and, on end user objectives and constraints. However, one main distinction can be made with, on the one side, reliable and detailed reconstruction approaches that rely on extensive and costly instrumentation, and on the other side, less detailed and precise, but more costeffective and lighter solutions. In this study, we have deliberately chosen to favor the second option, our opinion is that impact is higher with low-cost solutions.

Therefore, our aim is to provide methods to generate 3D models from existing buildings at reasonable costs and, relying only on available data. In particular, this implies not having to perform extensive data collection on the building itself. Hence our choice to focus on 3D models creation from 2D plans, which are available in most of the cases. Such methods are actually cost-effective but also less reliable: using building documentation can be error-prone and the quality of the 3D models will depend on the reliability of the plans. One possible way of dealing with this shortcoming is to combine the automatic generation of $3 \mathrm{D}$ models from 2D plans with focused manual intervention. The issue here is to minimize the time spent on manual corrections and maximize their impacts. In other words, the aim is to allow for assisted models correction.

The work presented in this paper aims at the development of tools for semiautomated, assisted 3D building model generation. This is an ongoing work, which follows up previous researches which lead to the development of a research prototype to automatically convert 2D scanned plans into a 3D building models [4]. The reconstruction is based on three components: geometry, topology and semantics. Geometry defines shape and dimensions, topology defines relations between features and semantics describes additional characteristics, such as room function. Complete and valid building models can be generated only if these three kinds of information can be extracted from the input data. The analysis of the results obtained with this first prototype shows that some errors and inconsistencies in 3D models cannot be solved automatically. We propose therefore to combine an automatic recognition process with punctual guided human interventions, in order to reach the best compromise between the $3 \mathrm{D}$ reconstruction cost and time on the one side, and the quality of the final result on the other side.

Some works have already attempted to involve users in the recognition process. In [5], the 3D reconstruction starts with a DXF file. The user can fix some problems in the original file and tune parameters of the detection algorithm before the automatic process is launched. Other solutions enable the user to delete some strings during text processing [6]. Some approaches have aimed at automatically interpreting and correcting architectural drawings while they are sketched. For instance, solutions based on a multi-agents system allow to recognize graphical objects such as lines or circles but also spoken annotations [7,8]. Another approach focused on errors that can be made by an architect during the design process and 
highlighted the importance of detecting and correcting errors in the early phases to avoid an error propagation [9]. However, to the best of our knowledge, no published work has dealt with the combination of automatic 3D generation and assisted correction, in the way the approach presented in this paper does.

In what follows, we first outline the framework that underlies our assisted model correction approach along three key components (section 2): (i) 3D generation errors taxonomy and sorting; (ii) 3D reconstruction rules; (iii) the generation process. Then we give an account of the experiments led with the research prototype we have developed and tested on 90 architectural floor plans (section 3).

\section{A framework for assisted 3D models correction}

\subsection{Error taxonomy and classification}

In previous steps of our research, we have developed a prototype to automatically convert a 2D scanned plan into a 3D building model [4]. One main outcome of this work is that automated transformation of a $2 \mathrm{D}$ paper plan into a valid and complete 3D building model is beyond reach. Some generation errors resulting from approximations in the plans itself, or from awkward interpretation of complex graphical patterns can only be solved thanks to human interpretation and intervention. For example, a missing topological link between two walls can have a strong impact on the correctness of the generated models but cannot be easily detected through automated processing.

In order to overcome this limitation, we have lead a thorough assessment of errors that can be found in generated models. Based on this assessment, we have then defined an error taxonomy structured along the three main components of 3D models: geometry, topology and semantics. In this taxonomy, for example, confusing a door with a window is labeled as a semantic error while a missing wall intersection is labeled as a geometrical and topological error.

In a second step, we have further analyzed the taxonomy to associate an impact score to each error. In this scope, we considered that the main application perspective was to use 3D building models for simulation purposes in the scope of renovation design. This led us to choose the four following criteria:

1. Impact on the energy simulation

2. Impact on the functional integrity of the building

3. Dependence of the error with other errors

4. Impact on the layout reconstruction

For each error, each item is given a score between 0 and 2 ( 0 if the error has no impact on the item and 2 if the error has a strong impact on the item). Then, a final score is calculated by summing individual scores to represent the overall criticality between 0 and 8 . For example, a missing window has a criticality of 4 , an open outer shape has a criticality equals to 8 . Table 1 gives an extract of the error classification framework.

The complete sorting shows that errors concerning the outdoor elements have a strong impact on the resulting 3D building model because all indoor elements 
Table 1: Extract of the classification errors table.

\begin{tabular}{|l|c|c|c|c|c|}
\hline Errors/impact & $\begin{array}{c}\text { Energy } \\
\text { simulation }\end{array}$ & $\begin{array}{c}\text { Functional } \\
\text { building integrity }\end{array}$ & $\begin{array}{c}\text { Dependence } \\
\text { with other errors }\end{array}$ & $\begin{array}{c}\text { Layout } \\
\text { reconstruction }\end{array}$ & Total \\
\hline Isolated wall & $0 / 2$ & $1 / 2$ & $1 / 2$ & $1 / 2$ & $3 / 8$ \\
\hline $\begin{array}{l}\text { Missing building element: } \\
\text { window }\end{array}$ & $2 / 2$ & $1 / 2$ & $1 / 2$ & $0 / 2$ & $4 / 8$ \\
\hline Open Outer Shape & $2 / 2$ & $2 / 2$ & $2 / 2$ & $2 / 2$ & $8 / 8$ \\
\hline
\end{tabular}

are recognized from them. This is why we deliberately set the focus on outdoor elements correction.

\subsection{Rules for outdoor element reconstruction}

During the automatic recognition process, rules are applied to detect outdoor elements. Outdoor elements are composed of walls and openings - each opening being a door or a window. The first step of the recognition process is to generate the outer shape of the building. The outer shape has to be closed and composed of outdoor walls. To fill topological constraints, each outdoor wall shall be connected with two other outdoor walls.

Several errors may occur, as listed below:

1. An open outer shape

2. Incomplete topology: an outdoor wall has only one or zero connection with other outdoor walls

3. Wrong adjustment between two walls

4. Missing walls

The semi-automatic process for error detection and correction developed in this paper has been elaborated according to the errors listed above. In the specific scope we are addressing (renovation construction), we focus on outdoor building elements. However, the same methodology can be generalized in a different context or applied on other building elements.

\subsection{Error detection and correction process}

Based on the above taxonomy, a system to detect inconsistencies in the generated building model has been developed. This system detects inconsistencies, converts them into errors and stores them. Depending on its criticality, an error can be treated directly or at the end of the outdoor elements recognition. For each error, several choices for correction are proposed to the user. These proposals are made according to the nature of the error and to topological or semantic considerations. Each choice and its consequence on the model is represented in an image. The user 
may then select the most appropriate image. At the end of the correction, the error list is updated. The semi-automatic process is over when the error list is empty or when it is not possible to propose any solution for all remaining errors.

Figure 1 shows an example of a user intervention when a topological connection is missing. Two outdoor walls (the black and the hatched) have just one topological connection. Because there are closed, the process will try to connect them. Four proposals are made to the user: these walls are not linked (1), the hatched wall is not an outdoor wall (2), the black wall is not an outdoor wall (3) and the two walls are linked and formed a unique wall (4). The user will then choose the most appropriate image (here the fourth). In order to help the user, the original image and the image representing the current status of the building are given. Then, automatic correction will be done and the error list will be updated. The correction of an error can result in the deletion of others errors. In this example, the topological connection will also close the outer shape of the building.

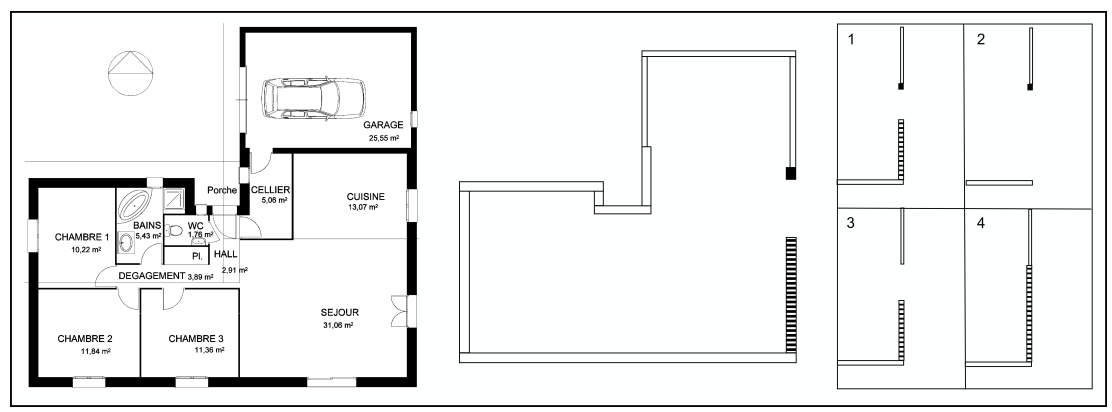

Figure 1: Example of an user intervention.

\section{Implementation, experiments and assessment}

\subsection{Implementation and experiments}

Our software to semi-automatically generate a 3D building model from a 2D scanned plan has been developed in $\mathrm{C}++$. The OpenCV library (Open Source Computer Vision Library) and functions developed by the Qgar project (http://www.qgar.org/) led in LORIA (France) have been used for image processing.

To evaluate our prototype, we used a database of original plan images from the "Universitat Autonoma de Barcelona" that has already been widely used [10]. This database includes 90 floor plans from various complexity, details and configurations. Figure 2 gives an example of an original floor plan used as input data in our validation and testing activities. 
Figure 3 represents the recognition results after the fully automated process (on the left) and the recognition results after the assisted correction process (on the right). Seven human interventions have been necessary to correct the model. In this example, the right part of the building has been well reconstructed by the automated process. However, in the left part, the geometrical shape is more complex and human interventions are needed. Automated recognition resulted into semantic confusions between indoor and outdoor walls. Human interventions allowed to correct these semantic errors, to create a new wall in the lower left-hand of the building and, to close the outer shape of the building.



Figure 2: Example of an original floor plan image.

In order to evaluate our results, we have considered the method presented in [10], which is based on a pixel-level evaluation. The idea is to compare results of the recognition using a ground-truth image pixel by pixel. A pixel can have only two values: 1 if it is a wall pixel or 0 . Then, the Jaccard Index (JI) is used to present results, it takes into account pixels that are: true positive, false positive and false negative.

$$
J I=\frac{\text { True Positive }}{\text { True Positive }+ \text { False Positive }+ \text { False Negative }}
$$

In our case, we do not just recognize walls but also openings and distinguish between indoor and outdoor elements. Therefore, in order to better evaluate our results, we have decided to create a new ground-truth image taking into account the following building elements: indoor \& outdoor walls, indoor \& outdoor doors and outdoor windows. We have manually annotated each plan of the database by distinguish each previously cited building elements. In this article, we focus only 


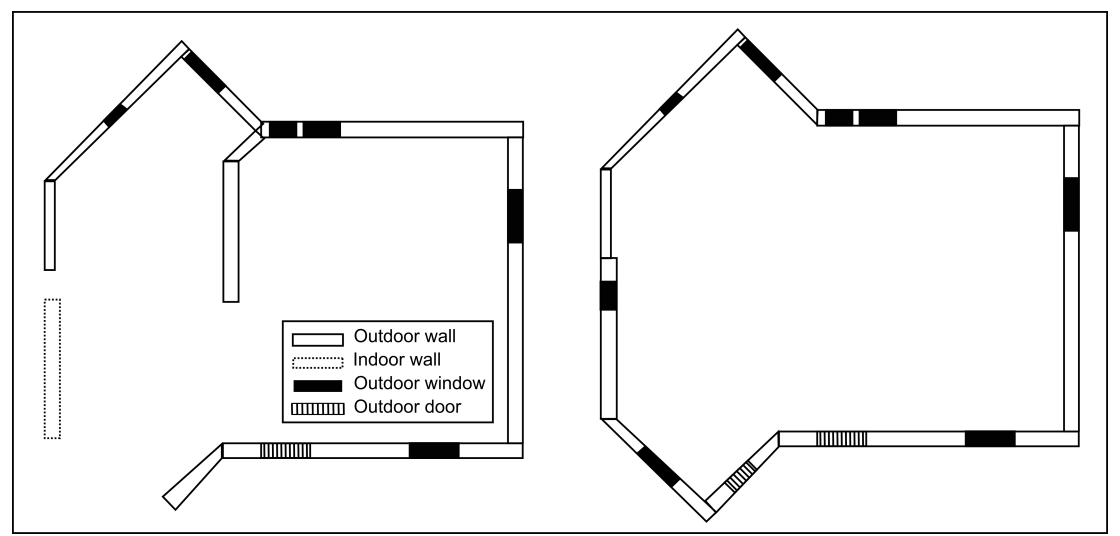

Figure 3: On the left, results of the automatic process and on the right, results of the semi-automatic process. Outdoor windows are represented with a black rectangle and outdoor door with a hatched rectangle.

on the outdoor element recognition and correction. This is the reason why we have evaluated our results using the Jaccard Index according to outdoor walls and outdoor openings. Results for the 90 floor plans are presented in the figure 4 .

The average Jaccard Index is 0.83 for outdoor walls, 0.75 for outdoor openings and 0.83 for the global outdoor element evaluation. The results are good given the fact that a little offset in an element position can have a high impact during the evaluation at pixel level. We have also calculated the number of well recognized outdoor openings: 989 out of $1083(91 \%)$ and the number of recognized linear meters of outdoor wall: $4573 \mathrm{~m}$ out of $4785 \mathrm{~m}(96 \%)$. However it is important to note the strong impact of walls in the recognition because in the figure 4 the global outdoor and the wall outdoor representations are very similar.

\subsection{Results assessment}

The aim of the semi-automatic process is to reduce the time spent to reconstruct a $3 \mathrm{D}$ building model. The figure 5 shows the frequency of the number of questions asked to the user per plan. It shows that for a large majority of plans, less than 15 actions are required. However, for some plans more than 40 actions are necessary. But these plans have the particularity to include some outdoor walls that are not part of the outer shape (for example, a swimming pool machine close to the house). The average of the number of questions is 13 for the evaluation of the 90 floor plans. For each action, the user has to choose between 4 to 6 proposal images by clicking on the most appropriate.

During the semi-automatic process, effects of each user action are stored in order to evaluate their impact on the 3D building model. The graphic in figure 6 summarizes effects of each action. Usually, a user interaction will produce around two different effects. A third of actions will cause no effect of the $3 \mathrm{D}$ building 


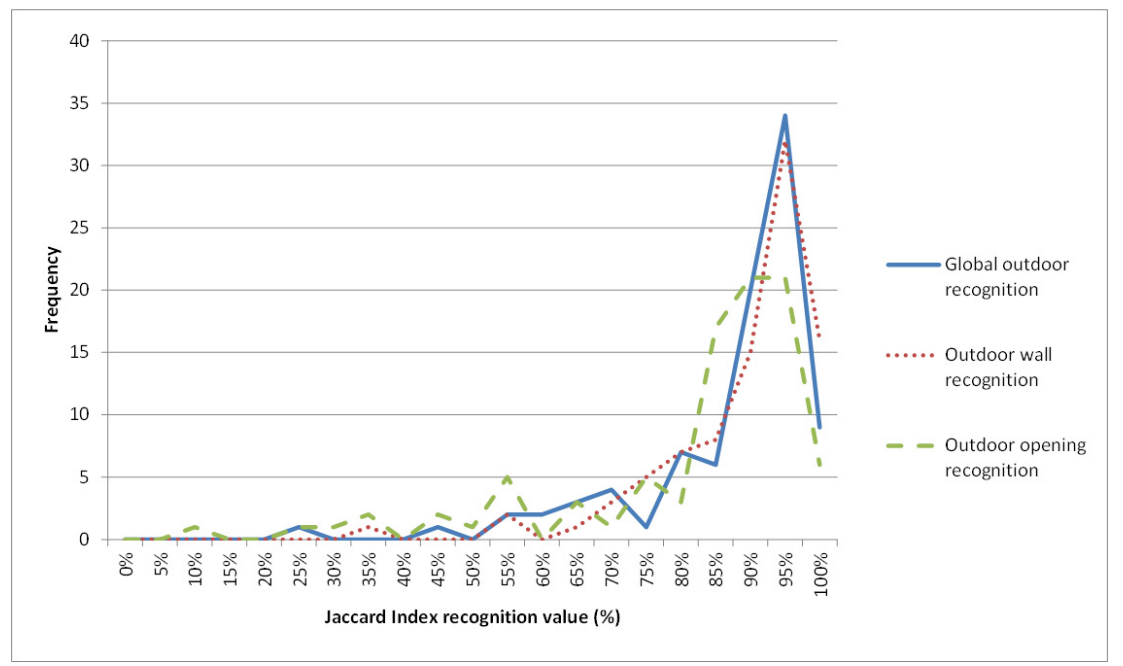

Figure 4: Frequency of the Jaccard Index recognition value applied to outdoor wall and outdoor opening recognition for the 90 floor plans.

model, it corresponds to cases where the two elements implied in the proposition are not linked. Then, the most important effects are wall modifications and the deletion of errors. Sometimes an action can lead to another question when complementary information is needed. For example, when a wall is missing, it can be necessary to specify its position related to existing walls. Usually the first actions have a stronger effect on the recognition than the others, due to the error classification and notation.

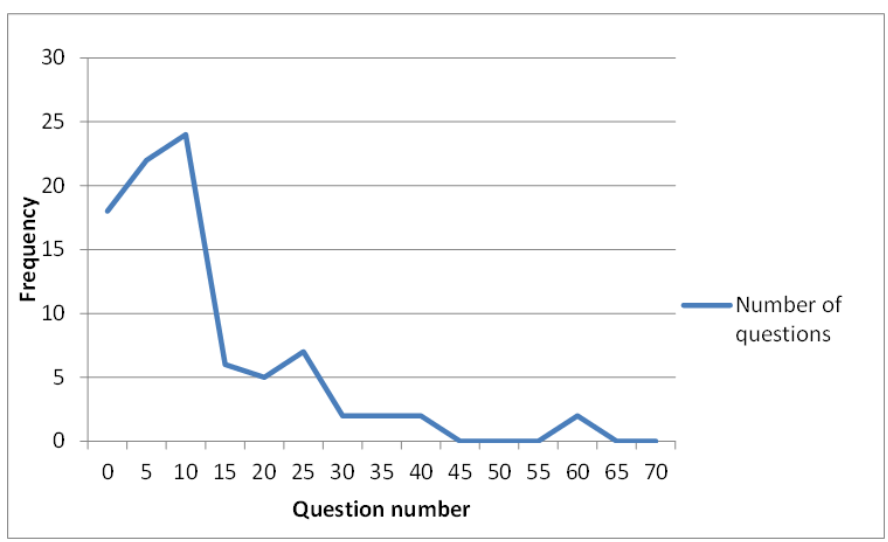

Figure 5: Frequency of the number of questions asked to the user for the 90 floor plans. 


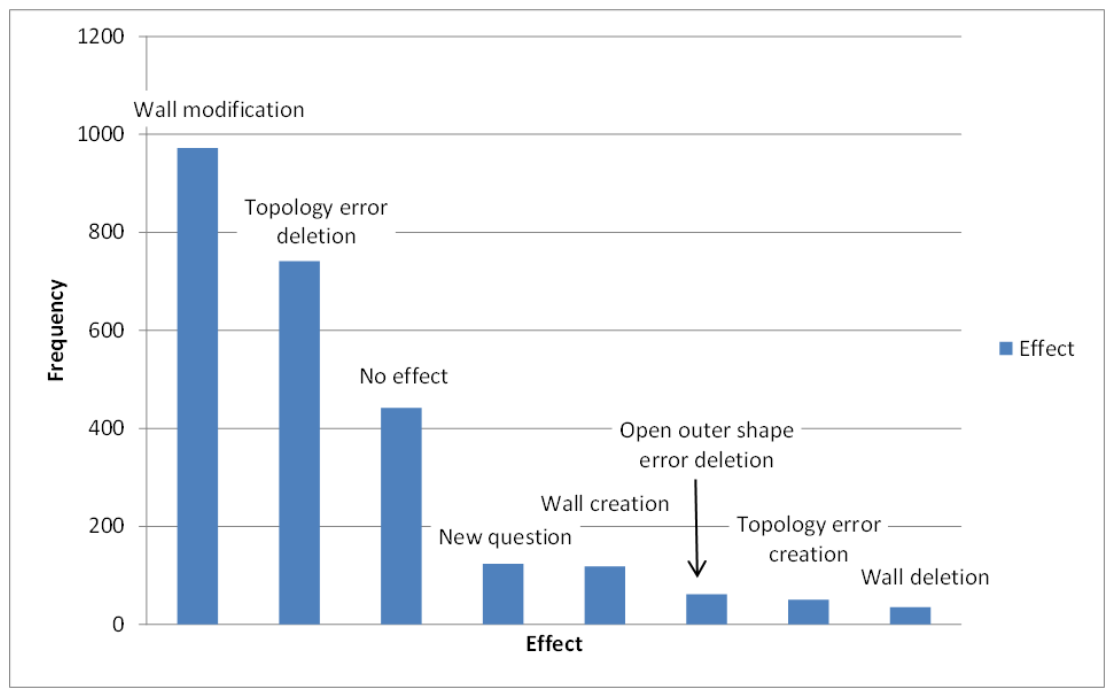

Figure 6: Distribution of the effect after a user action decision.

\subsection{Discussion}

The combination of an automatic recognition process with punctual and guided human interventions allows to obtain good results for the outdoor element identification and recognition. Due to the modification of walls, many errors like open outer shape or missing topological connections are corrected. One action of the user can cause several effects on the resulting 3D building model. Our method based on the correction of an error according to its criticality allows to avoid the propagation of an error and to propose appropriate proposals to limit the number of interactions.

This tool has been developed to be available to non-expert building users. This is why, actions asked are simple and consisted in clicking on the good image. With this correction process, the user can directly visualize effect of its choice on the building before choosing. A promising improvement could be to develop a process to sort proposals according to their relevance to help even more the user.

The evaluation by pixel is a good way to obtain a global result of the recognition quality. However, during the recognition, some rules are applied to adjust some walls according to topological characteristics and these processes can cause some little offsets in the building element position. This is why, an evaluation by counting the number of well recognized openings or of well recognized linear meters of walls is good complementary result to better quantify the recognition quality.

Still, some improvements can be done. For some plans, too many actions are necessary (more than 40). This happens when building have atypic outer shape 
(with an additional room close to the house for example) or when more than one wall is missing between two walls. This case is also difficult to automatically correct. One solution could be to ask the user to redraw some missing elements of the building. This new action could allow to improve the recognition result without spending a lot of time because just some elements are missing.

Moreover, the process has been tested only for the outdoor element recognition. The next step is to develop the same methods and process to the semi-automatic recognition of indoor elements.

Some information is missing on the $2 \mathrm{D}$ scanned plan like the third dimension: heights of the building and openings for example. To get the exact measurements, a solution is to use results from the research done on building facade images processing [11] or to do some punctual data acquisition on-site.

\section{Conclusion}

The paper describes the results of an ongoing research work that aims to develop a prototype to semi-automatically convert a 2D scanned plan into a 3D building model. The aim is to effectively combine au automated 3D model generation software developed in earlier phases with an assisted correction approach, to enhance the quality of the generated models. During the building elements recognition, errors are detected in the $3 \mathrm{D}$ building model and candidate corrections are proposed to the user. This process based on punctual and guided human interventions allows to reach a good compromise between the time spent to the 3D building model generation, the cost of the reconstruction, and the reliability of 3D models. An evaluation made thanks to a database of 90 floor plans has given encouraging results and shows the benefits of human interventions. Future works will be focused on the integration of error detection and correction for indoor building elements using the same methodology. Another perspective is to supplement 2D plans with additional sources of data (like facade image segmentation) to improve the 3D building model.

\section{Acknowledgement}

The work presented in this paper is part of the HOLISTEEC project (Grant Agreement 609138), funded by the European Commission under the FP7 program (Energy-Efficient Building Public-Private Partnership).

\section{References}

[1] Juan, Y.K., Gao, P. \& Wang, J., A hybrid decision support system for sustainable office building renovation and energy performance improvement. Energy and Buildings, 42(3), pp. 290-297, 2010.

[2] Volk, R., Stengel, J. \& Schultmann, F., Building information modeling (BIM) for existing buildings - literature review and future needs. Automation in Construction, 38(0), pp. 109-127, 2014. 
[3] Gimenez, L., Hippolyte, J.L., Robert, S., Suard, F. \& Zreik, K., Review: reconstruction of $3 \mathrm{~d}$ building information models from $2 \mathrm{~d}$ scanned plans. Journal of Building Engineering, 2(0), pp. 24-35, 2015.

[4] Gimenez, L., Robert, S., Suard, F. \& Zreik, K., Reconstruction of 3d building models from $2 \mathrm{~d}$ scanned plans-opening the path for enhanced decision support in renovation design. ECPPM 2014, eWork and eBusiness in Architecture Engineering and Construction, pp. 75-80, 2014.

[5] Dominguez, B., Garcia, A. \& Feito, F., Semiautomatic detection of floor topology from CAD architectural drawings. Computer-Aided Design, 44, pp. 367-378, 2012.

[6] Dosch, P., Tombre, K., Ah-Soon, C. \& Masini, G., A complete system for the analysis of architectural drawings. International Journal on Document Analysis and Recognition, pp. 102-116, 2000.

[7] Azar, S., Couvreur, L., Delfosse, V., Jaspart, B. \& Boulanger, C., An agentbased multimodal interface for sketch interpretation. Multimedia Signal Processing, 2006 IEEE 8th Workshop on, pp. 488-492, 2006.

[8] Fernandez-Pacheco, D., Albert, F., Aleixos, N. \& Conesa, J., A new paradigm based on agents applied to free-hand sketch recognition. Expert Systems with Applications, 39, pp. 7181-7195, 2012.

[9] Safin, S., Leclercq, P. \& Blavier, A., Errors in architectural design process: towards a cognitive model. International Design Conference-Design 2008, Dubrovnik, Croatia, 2008.

[10] Heras, L.P., Ahmed, S., Liwicki, M., Valveny, E. \& Sánchez, G., Statistical segmentation and structural recognition for floor plan interpretation. International Journal on Document Analysis and Recognition (IJDAR), pp. $1-17,2013$.

[11] Ok, D., Kozinski, M., Marlet, R. \& Paragios, N., High-level bottom-up cues for top-down parsing of facade images. Second Joint 3DIM/3DPVT Conference: $3 D$ Imaging, Modeling, Processing, Visualization et Transmission, pp. 128-135, 2012. 